\title{
Reduction of Transmission Losses based on Optimal Power Flow using Genetic Algorithm
}

\author{
Jinendra Rahul $^{* 1}$, Yagvalkya Sharma ${ }^{\# 2}$, Dinesh Birla ${ }^{* 3}$ \\ *Electrical Engg. Department, University College of Engineering, RTU. \\ jinendra.regmail.com \\ ${ }^{3}$ birla.eckegmail.com.

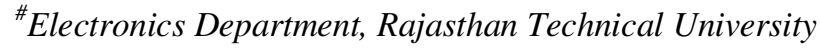 \\ 'yagvalkyaegmail.com
}

\begin{abstract}
This paper presents the application of Genetic Algorithm (GA) for solving optimal power flow problems. It is an important tool for performance analysis of many power systems problems. Optimal power flow (OPF) is of very much significance in power system operation analysis under deregulated environment of electricity industry. The OPF optimizes a power system operating objective function, while satisfying a set of system operating constraints. The basic OPF solution is obtained with production cost minimization as the objective function and the optimal settings of the power system are determined. OPF can also be formulated for reactive power optimization, as minimization of system active power losses and improving the voltage stability in the system. In the present paper objective function is to reduce transmission losses using GA, a IEEE 30-bus test power system is studied for optimal power flow. It is described in the paper that GA based optimal power flow can provide optimal solution.
\end{abstract}

Keywords - Genetic algorithm (GA), optimal power flow (OPF).

\section{INTRODUCTION}

The OPF problem was introduced in the early 1960s by Carpentier and has grown into a powerful tool for power system operation and planning. Since 1962, many algorithms have been designed to solve base OPF and its derivative problems [1].

As the development of power systems and computing technologies, OPF formulation becomes more and more complicated, large-scale, and realistic. OPF has been widely used in power system operation and planning.

Many optimization techniques have been applied to solve OPF problems, such as mathematical programming (such as linear programming [1], quadratic programming [2], dynamic programming [3], gradient methods and Lagrangian relaxation approaches) and modem meta-heuristic methods (such as simulated annealing, genetic algorithms [4],

Dinesh Birla is Associate Professor in the Department of EE, University College of Engg., Rajasthan Technical University, Kota.

Jinendra Rahul and Yagvalkya Sharma are PG students in the University College of Engg., Rajasthan Technical University, Kota. evolutionary algorithms [5], adaptive tabu search, particle swarm optimization etc.). Some of these methods are successful in locating the optimal solution, but they are usually slow in convergence and require very expensive computational cost. Some other methods may risk being trapped to a local optimum, which is the problem of premature convergence.

In this paper, an application of Genetic Algorithm to reduce transmission losses using optimal power flow is proposed. The controllable system quantities are generator power MW, controlled voltage magnitude, reactive power injection from reactive power sources and transformer tap setting. The objective used herein is to minimize the total transmission loss by optimizing the control variables within their limits. Therefore, no violation on other quantities (e.g. MVA flow of transmission lines, load bus voltage magnitude, generator MVAR) occurs in normal system operating conditions. The proposed method has been tested on IEEE 30-bus test power system.

In this paper, the formulation of optimal power flow is explained in Section 2 in such a way that the total transmission loss of system is employed to be the system objective. Section 3 provides a brief of Genetic Algorithm to solve non-linear optimization problems. The IEEE 30-bus test power system was challenged and therefore discussed in Section 4. The last section provides the conclusions.

\section{OPTIMAL POWER FLOW PROBLEM}

The OPF problem has grown into a powerful tool for power system operation and planning. In general, the optimal power flow problem is a nonlinear optimization problem. It is used to minimize a desired objective function, subject to certain system constraints [8]. 
The general optimal power flow problem can be expressed as a constrained optimization problem as follows,

\section{Minimize $\quad f(x)$}

Subject to- $\quad g(x)=0$, equality constraints $h(x) \geq 0$, inequality constraints

A. Objective function

The objective function for total power transmission loss can be expressed as follows,

$F_{\text {Tloss }}=\sum_{i=1}^{N L} \sum_{j=1}^{N_{L}} g_{i, j}\left\{V_{i}^{2}+V_{j}^{2}-2 V_{i} V_{j} \cos \left(\delta_{i}-\delta_{j}\right)\right\}$

Where,

$V_{i}=$ the voltage magnitude at bus $i$.

$N_{L}=$ the total number of transmission lines.

$\delta_{i}=$ the voltage angle at bus $i$.

$g_{i, j}=$ the conductance of line $i-j$.

\section{B. System Constraints}

These are system constraints to be formed as equality and inequality constraints as shown below [6].

1) Equality constraint: Power flow equations are,

For Real Power balance eq.,

$$
\begin{gathered}
P_{G i}-P_{D i}-\sum_{j=1}^{N_{B}}\left|V_{i}\right|\left|V_{j}\right|\left|Y_{i, j}\right| \cos \left(\theta_{i, j}-\delta_{i}+\delta_{j}\right) \\
=0
\end{gathered}
$$

For Reactive Power balance eq.,

$$
Q_{G i}-Q_{D i}+\sum_{j=1}^{N_{B}}\left|V_{i}\right|\left|V_{j}\right|\left|Y_{i, j}\right| \sin \left(\theta_{i, j}-\delta_{i}+\delta_{j}\right)
$$

$$
=0
$$

Where,

$$
i=1,2,3, \ldots \ldots \ldots \ldots N_{\text {bus }-1} \text {. }
$$

$P_{G i}=$ the real power generation at bus i.

$Q_{G i}=$ the reactive power generation at bus $\mathrm{i}$.

$P_{D i}=$ the real power demand at bus $i$.

$Q_{D i}=$ the reactive power demand at bus $i$.

$N_{B}=$ the total number of buses.

$\theta_{i, j}=$ the angle of bus admittance element $\mathrm{i}, \mathrm{j}$.
$Y_{i, j}=$ the magnitude of Ybus element $\mathrm{i}, \mathrm{j}$.

2) Inequality constraint: Variable limitations are,

$$
\begin{gathered}
V_{i}^{\min } \leq V_{i} \leq V_{i}^{\max } \\
T_{i}^{\text {min }} \leq T_{i} \leq T_{i}^{\max } \\
Q_{\text {comp }, i}^{\min } \leq Q_{\text {comp }, i} \leq Q_{\text {comp }, i}^{\max } \\
P_{G, i}^{\min } \leq P_{G, i} \leq P_{G, i}^{\max }
\end{gathered}
$$

Where,

$V_{i}^{\min }, V_{i}^{\max }=$ upper and lower limits of voltage magnitude at bus $i$.

$T_{i}^{\min }, T_{i}^{\max }=$ upper and lower limits of tap position of transformer i.

$Q_{c o m p, i}^{\min }, Q_{c o m p, i}^{\max }=$ upper and lower limits of reactive power source $i$.

$P_{G, i}^{\min }, P_{G, i}^{\max }=$ upper and lower limits of power generated by generator $i$.

The penalty function can be formulated as follows,

$$
P(x)=F_{T}+\Omega_{P}+\Omega_{Q}+\Omega_{C}+\Omega_{T}+\Omega_{V}+\Omega_{G}
$$

Where,

$$
\begin{aligned}
& \Omega_{P}=\rho \sum_{i=1}^{N_{B}}\left\{P_{G, i}-P_{D, i}\right. \\
& -\sum_{j=1}^{N_{B}}\left|V_{i}\right|\left|V_{j}\right|\left|Y_{i j}\right| \cos \left(\theta_{i, j}-\delta_{i}\right. \\
& \left.\left.+\delta_{j}\right)\right\}^{2} \\
& \Omega_{Q}=\rho \sum_{i=1}^{N_{B}}\left\{Q_{G, i}-Q_{D, i}\right. \\
& -\sum_{j=1}^{N_{B}}\left|V_{i}\right|\left|V_{j}\right|\left|Y_{i j}\right| \cos \left(\theta_{i, j}-\delta_{i}\right. \\
& \left.\left.+\delta_{j}\right)\right\}^{2} \\
& \Omega_{C}=\rho \sum_{i=1}^{N_{C}}\left\{\max \left(0, Q_{c o m p, i}-Q_{c o m p, i}^{\max }\right)\right\}^{2} \\
& +\rho \sum_{i=1}^{N_{C}}\left\{\operatorname { m a x } \left(0, Q_{c o m p, i}^{\min }\right.\right. \\
& \left.\left.-Q_{\text {comp }, i}\right)\right\}^{2}
\end{aligned}
$$




$$
\begin{array}{r}
\Omega_{T}=\rho \sum_{i=1}^{N_{T}}\left\{\max \left(0, T_{i}-T_{i}^{\max }\right)\right\}^{2} \\
+\rho \sum_{i=1}^{N_{T}}\left\{\max \left(0, T_{i}^{\text {min }}-T_{i}\right)\right\}^{2} \\
\Omega_{V}=\rho \sum_{i=1}^{N_{B}}\left\{\max \left(0, V_{i}-V_{i}^{\max }\right)\right\}^{2} \\
+\rho \sum_{i=1}^{N_{B}}\left\{\max \left(0, V_{i}^{\min }-V_{i}\right)\right\}^{2}
\end{array}
$$

$$
\begin{aligned}
\Omega_{G}=\rho \sum_{i=1}^{N_{G}}\left\{\max \left(0, P_{G, i}-P_{G, i}^{\max }\right)\right\}^{2} & \\
& +\rho \sum_{i=1}^{N_{G}}\left\{\max \left(0, P_{G, i}^{\min }-P_{G, i}\right)\right\}^{2}
\end{aligned}
$$

Where,

$\mathrm{N}_{\mathrm{G}}=$ the total number of generators.

$\mathrm{N}_{\mathrm{C}}=$ the total number of reactive power sources.

$\mathrm{N}_{\mathrm{T}}=$ the total number of transformers.

\section{GENETIC ALGORITHM}

Genetic algorithm (GA) is a global adaptive search technique based on the mechanics of natural genetics. GA uses a direct analogy of natural behavior. It is applied to optimize existing solutions by using methods based on biological evolution such as the ones presented by Charles Darwin. It has many applications in certain types of problems that yield better results than the commonly used methods without any complicated classical calculation. To solve a specific problem with GA, a function known, as fitness function needs to be constructed which allows different possible solutions to be evaluated. The algorithm will then take those solutions and evaluate each one, deleting the ones that show no promise towards a result but keeping those, which seem to show some activity towards a working solution [9].

For this paper, continuous GA is used due to it has an advantage in term of accuracy representation of continuous parameter. In this paper, the GA is selected to build up an algorithm to solve optimal power flow problems (all generation from available generating units). To reduce programming complication, the Genetic Algorithm (GADS TOOLBOX in MATLAB) is employed to generate a set of initial random parameters. With the searching process, the parameters are adjusted to give the best result [10].

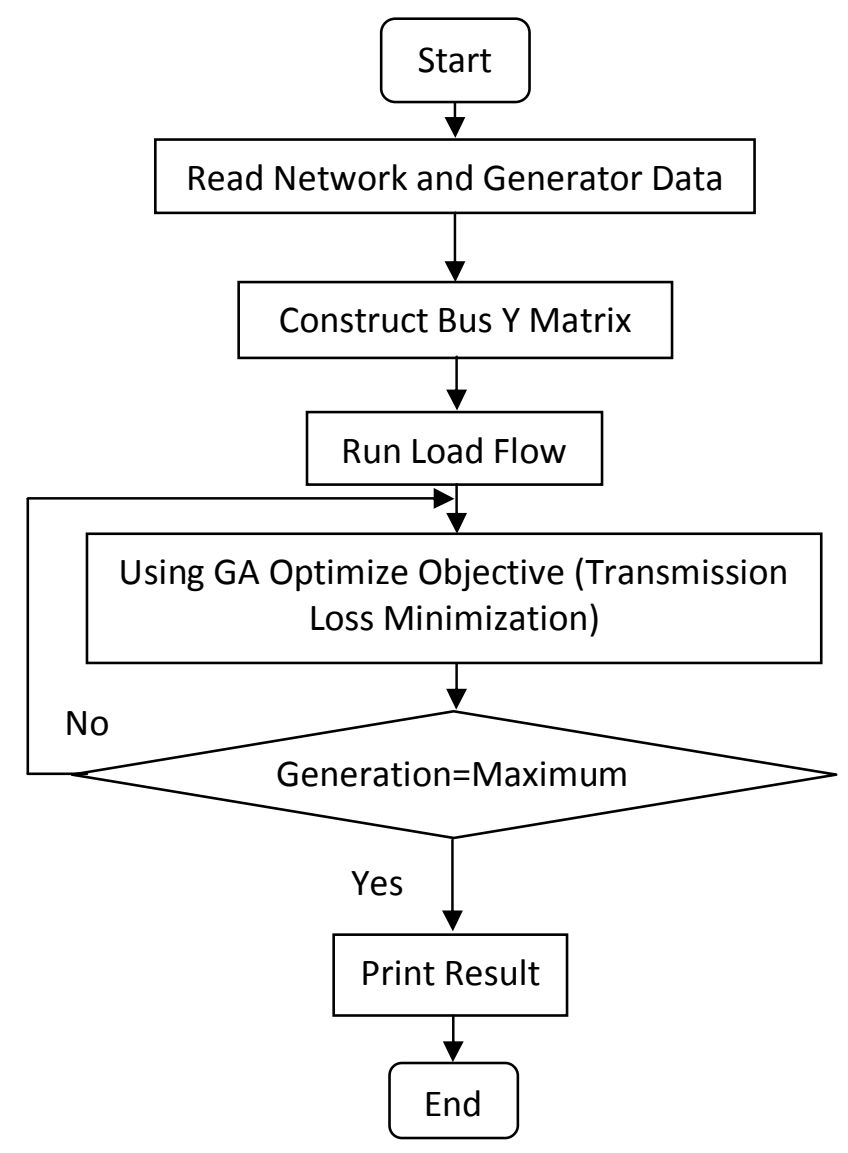

Figure 1. Flowchart of GA application to transmission loss

\section{SIMULATION RESULTS}

In this paper, a IEEE 30-bus test power system as given in Fig. 2 was chosen as a test system. The Quasi-Newton with BFGS Formula, Particle Swarm Optimization and Genetic Algorithms were employed for comparison. Each method was carried out by 30 trials. Table 1 gave limitations of variables used for Optimal Power Flow.

TABLE I

LIMITS OF CONTROL VARIABLES UESED FOR OPTIMAL POWER FLOW

\begin{tabular}{|c|c|c|}
\hline Variables & Lower limit & Upper limit \\
\hline$P_{G 1}(M W)$ & 220 & 300 \\
\hline$P_{G 2}(M W)$ & 20 & 60 \\
\hline V1-V30 (p.u.) & 0.9 & 1.1 \\
\hline$T_{1}($ Mvar $)$ & 0.9 & 1.1 \\
\hline$T_{2}($ Mvar $)$ & 0.9 & 1.1 \\
\hline$Q_{G 1}($ Mvar $)$ & -25 & -10 \\
\hline$Q_{G 2}($ Mvar $)$ & 40 & 60 \\
\hline$Q_{G 3}($ Mvar $)$ & 30 & 40 \\
\hline$Q_{G 4}($ Mvar $)$ & 20 & 35 \\
\hline$Q_{G 5}($ Mvar $)$ & 10 & 20 \\
\hline$Q_{G 6}($ Mvar $)$ & 5 & 15 \\
\hline \multicolumn{2}{|c|}{} \\
\hline
\end{tabular}

To find a set of optimal power flow solution, the proposed method, Genetic Algorithm was employed. 
For benchmarking, the Quasi-Newton method and Particle Swarm Optimization were also employed.

TABLE II

SIMULATION RESULTS OF IEEE 30-BUS TEST POWER SYSTEMS

\begin{tabular}{|c|c|}
\hline & $\begin{array}{c}\text { Power Transmission Losses } \\
\text { (MW) }\end{array}$ \\
\hline Quasi-Newton with BFGS & 16.16 \\
\hline PSO & 13.55 \\
\hline GA & 12.832 \\
\hline
\end{tabular}

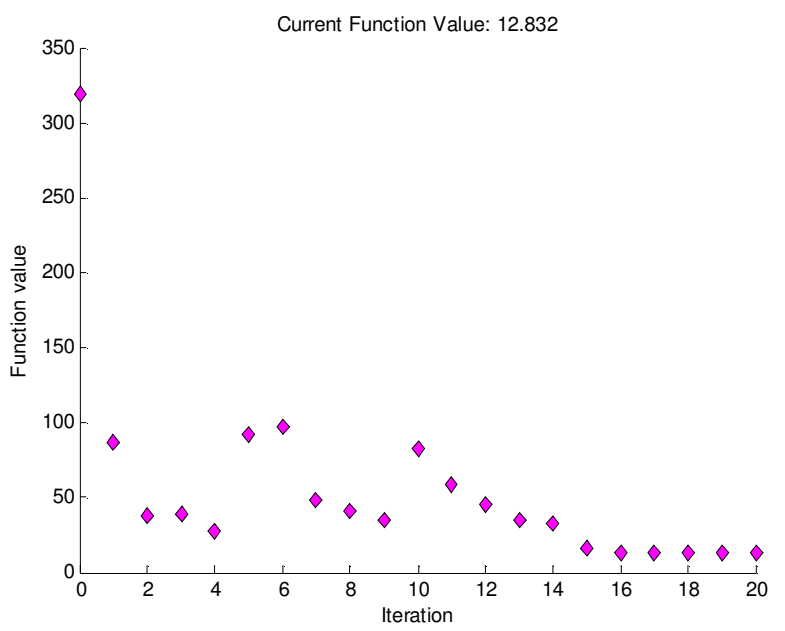

Figure 2. Function value characteristic using GA.

For 30-bus test system the average power loss solutions are 40.20 MW, $17.72 \mathrm{MW}$ and 12.832 MW for the BFGS, GA and PSO methods, respectively. This reveals that the GA method is the most efficient method among these three methods of solving the optimal power flow problem with the power loss objective.

\section{CONCLUSIONS}

This paper described the use of Genetic Algorithm to find optimal power flow solutions. This work was conducted by 30 trials. The test also applied the Quasi-Newton method and Particle Swarm Optimization of 30 trials each for comparison. The results showed that a set of optimal solutions with respect to the power transmission loss objective can be efficiently found. As a result, the GA method proves that it can find a place among some efficient search methods in order to find a near global solution of the optimal power flow problems.

\section{REFERENCES}

[1] B. Stott and J. L. Marinho, "Linear programming for power system network security applications", IEEE Transactions on Power Apparatus and Systems, Vol. PAS-98, pp. 837-848, 1979.

[2] G. F. Reid and L. Hasdorf, "Economic dispatch using quadratic programming", IEEE Transactions on Power Apparatus and Systems, Vol. PAS-92, pp. 2015-2023, 1973.
[3] H. W. Dommel and W. F. Tinney, "Optimal power flow solutions", IEEE Transactions on Power Apparatus and Systems, Vol. PAS-87, pp. 1866-1876, October 1968.

[4] G. Bakirtzis, N. Biskas, E. Zoumas and Vasilios Petridis, "Optimal power flow by enhanced genetic algorithm", IEEE Transactions on Power Systems, Vol.17, pp. 229-236, 2002.

[5] Jason Yuryevich and Kit Po Wong, "Evolutionary programming based optimal power flow algorithm”, IEEE Transactions on Power Systems, Vol.14, pp. 1245-1250, 1999.

[6] A.J. Wood and B.F. Wollenberg, "Power generation, operation and control”, Wiley-Interscience, New York, 1996.

[7] William D. Rosehart, Victor H. Quintana and Claudio A. Cañizares, "Multiobjective Optimal Power Flows to Evaluate Voltage Security Costs in Power Networks", IEEE Transactions on Power Systems, Vol. 18, No. 2, May 2003.

[8] Kyung II Min, Sang-Hyeon Ha, Su-Won Lee,and Young-Hyun Moon, "Transmission loss allocation algorithm using path-integral based on transaction strategy", IEEE Transaction on power systems, vol.25, pp.195-205, Feb.,2010.

[9] Z. Haibo, Z. Lizi and M. Fanling, "Reactive power optimization based on genetic algorithm”, International Power Conference on Power System Technology, pp.1448 - 1453, 18-21 August 1998.

[10] Mohd Herwan Sulaiman, Mohd Wazir Mustafa and Omar Aliman, "Transmission Loss and Load Flow Allocations via Genetic Algorithm Technique", From IEEE Xplore, TENCON 2009. 